\title{
Entrevista
}

\section{Albert Sangrà, \\ convencer con \\ evidencias}

DOI: 10.29236/sistemas.n158a3

Es su máxima cuando se refiere al potencial de la educación en línea bien diseñada, para fortalecerla en todo el mundo.

\section{Sara Gallardo M.}

Desde muy niño a orillas del mar Mediterráneo, Albert Sangrà supo que la educación era su norte para ser feliz. De ahí que recuerde con tanto orgullo sus inicios como maestro de educación primaria, seguidos de tanto estudio a través de los años.

"Soy doctor en Pedagogía por la Universitat Rovira i Virgili de Tarragona (España), pero empecé siendo licenciado en Filosofía y Ciencias de la Educación, en la Universitat de Barcelona, para seguir con un posgrado en Applications of ICT in Open and Distance Education, de la Open University del Reino Unido, además de un diplomado en Strategic Use of IT in Education de Harvard University", relata sin aspavientos.

Crónica que contempla la formación de directivos en la administración pública de Cataluña. "Me incorporé como profesor asociado de Pedagogía a la Universitat Ramon Llull y en 1995 fui llamado a formar parte del equipo fundador de la 


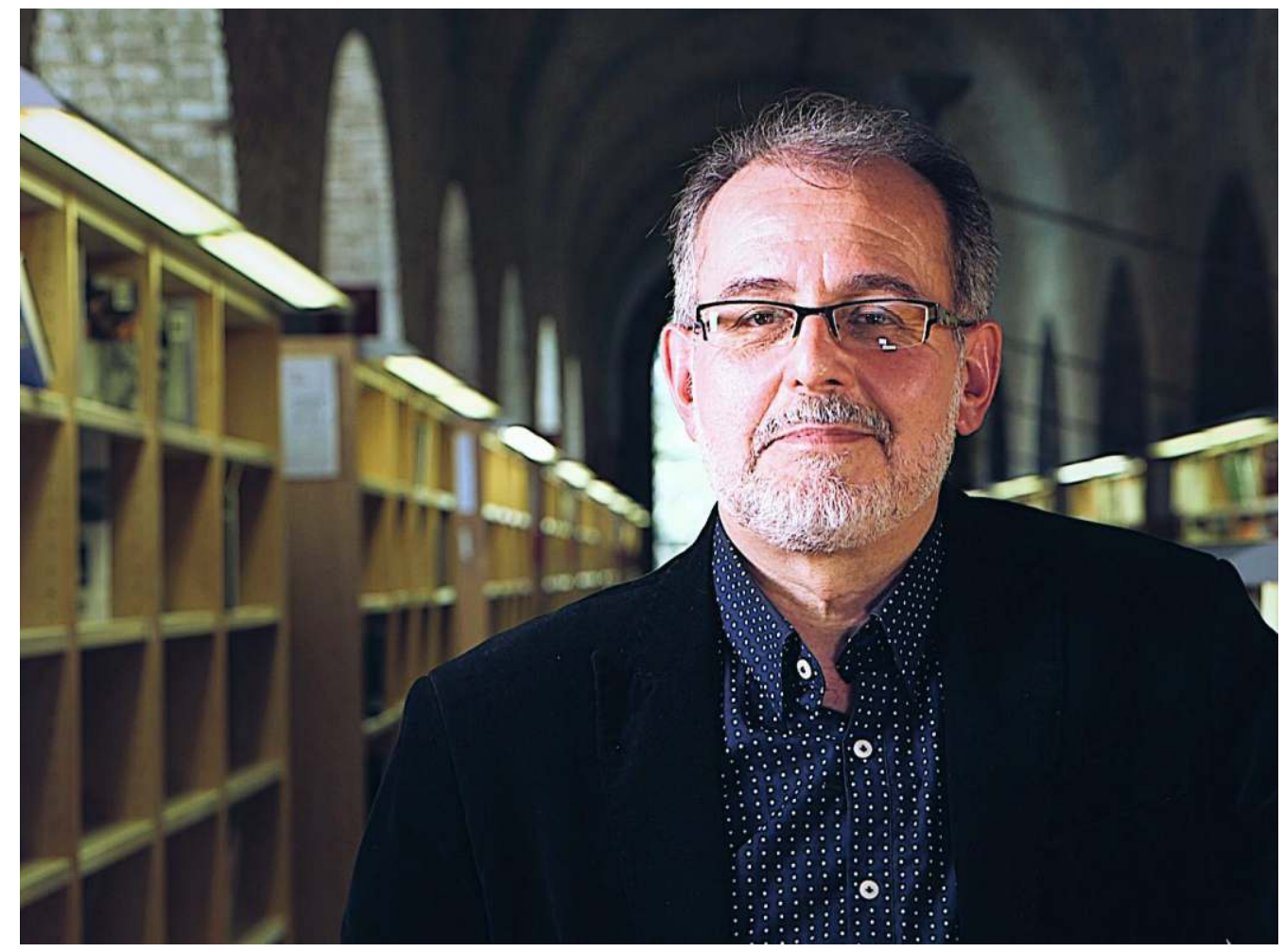

Universitat Oberta de Catalunya (UOC)".

Y ni qué decir de su trabajo en la Or-

danía del mundo. UNESCO es un excelente altavoz para poder llevarlo a cabo", asegura Albert Sangrà.

ganización de las Naciones Unidas para la Educación, la Ciencia y la Cultura (UNESCO). "Mi relación con esta entidad es muy pequeña comparada con la de muchísimas personas que trabajan sobre el terreno en muchos lugares, a quienes respeto y admiro muchísimo. Me siento muy honrado en mi modesta posición, aportando desde la Cátedra UNESCO en Educación y Tecnología para el Cambio Social de la Universitat Oberta de Catalunya, el trabajo, las inquietudes y los resultados de nuestra investigación y práctica, al conjunto de la ciuda-

Con la misma gratitud sigue repasando su historia. "He tenido la enorme suerte de poder participar en muchas iniciativas internacionales, además de compartir espacios y preocupaciones con personalidades muy relevantes de este campo, como los profesores Tony Bates, Michael G. Moore, Alan Tait, con quienes continúo colaborando y se han convertido en amigos. No quiero olvidar a tantas otras personas de las que he aprendido mucho a lo largo de mi trayectoria ni a los compañeros y compañeras que 
han caminado a mi lado durante todos estos años. A todos debo darles las gracias por su amistad y apoyo, en especial a los miembros del grupo de investigación EduI@b".

El cúmulo de recuerdos está acompasado con las notas del piano que intenta aprender a tocar, entre los acordes de la docencia y la investigación, sus más grandes pasiones.

Después de transitar por su ejercicio profesional, entramos en materia.

Revista Sistemas: Desde la entidad que usted representa ¿qué alcance tiene la educación del futuro? ¿Ejerce una influencia en los distintos Gobiernos, en el marco de la incertidumbre que genera el COVID-19?

Albert Sangrà: Tenemos que distinguir entre la UNESCO como organismo internacional, que tiene sus políticas generales respecto a la educación, y las Cátedras UNESCO que están distribuidas por el mundo. Estas Cátedras, a menudo bajo el paraguas institucional de una universidad, son especializadas en un ámbito concreto de la educación, y llevan a cabo sus propios programas y tienen su propia agenda, eso sí, alineada con los objetivos generales del organismo internacional $y$, concretamente hoy en día, también con la Agenda 2030, compartida no solo por dicha entidad, sino por otros organismos supranacionales.
La capacidad de poder influir en los distintos Gobiernos es muy variable, y depende del posicionamiento de la cátedra en cada país, pero también del índice de desarrollo del mismo, y de las condiciones que puedan darse para que esa influencia pueda llevarse a cabo. Lo que sí está claro, es que la mayoría de las cátedras elaboran estudios, señalan tendencias, y emiten recomendaciones para que las distintas organizaciones educativas, administraciones y Gobiernos puedan tomar decisiones mejor informadas.

RS: ¿Cómo observa los países en el mundo dentro de este contexto? ¿Cuáles considera son los riesgos y cuáles las oportunidades?

AS: La pandemia ha tensado las costuras de la educación en todo el mundo. En muchos casos, hemos visto como la historia del emperador que iba desnudo se ha convertido en realidad. Han emergido muchas carencias de la educación tradicional que hasta ahora no salían a la luz, simplemente porque se corría un tupido velo sobre ellas.

Por supuesto, los alumnos de los países menos desarrollados económica y socialmente son los más perjudicados, como ocurre siempre.

La interrupción de la educación ha sobrevenido en todos los sistemas educativos, y solo la adopción de la docencia remota, en algunos casos, y de la educación online en otros ha permitido que algunos pu- 
diesen superar esta situación de formas más o menos satisfactorias. Esto ha puesto de manifiesto que el uso de la tecnología en la educación ya no debe ser solo una cosa para países ricos; la equidad y la justicia en la educación pasan porque todas las personas puedan alfabetizarse digitalmente $y$, de esa forma acceder a las oportunidades de aprendizaje que otros ya tienen a su alcance. Ahí los Gobiernos y las administraciones, pero toda la población también, tienen una gran responsabilidad para encarar el futuro de la educación de forma más justa y equitativa.

Y por supuesto que hay riesgos en eso. Tenemos que evitar que el acceso al uso de la tecnología en educación sea solo coto de las empresas tecnológicas. Tenemos que desarrollar políticas que protejan el ingente tráfico de datos personales, y tenemos que desarrollar políticas y estrategias que no solo hagan Ilegar conectividad y dispositivos a las personas, sino que las capaciten y las empoderen para poder utilizarlas adecuadamente en procura de su desarrollo personal y profesional.

RS: Hay quienes manifiestan que la educación estará cifrada en las habilidades personales de cada individuo, más allá de los títulos obtenidos. ¿Qué opina al respecto?

AS: En realidad eso siempre ha sido así. Los títulos solo eran una certificación de las habilidades obtenidas. Hoy día la educación se ha convertido en un manjar muy jugoso también para aquellos que creen que, a través de ella, pueden ganar mucho dinero. Existe una importante campaña para desmerecer los títulos que ofrecen las universidades y hacer que estos títulos los ofrezcan las propias empresas o instituciones que tendrán en ese nicho su negocio. No digo que no pueda ser lícito. Lo que pasa es que tenemos que ser veraces. Las universidades garantizan que, junto a las capacidades instrumentales que las personas deban adquirir en un momento determinado, también adquieran competencias en pensamiento crítico, en pensamiento independiente, que hagan que no se limiten a reproducir un procedimiento, sino que sean capaces de valorar no solo si se puede mejorar, sino también si es ético y justo. Soy consciente de que, a pesar de eso, el mundo empresarial está decidido a intentar esta "opa hostil" a la independencia de la universidad $y$, en general, de la educación superior.

Por eso, la universidad también debe cambiar y convertirse en el nodo de calidad y de confianza para las personas que desarrollan una suerte de red personal para el aprendizaje.

RS: También se dice que las clases estarán dirigidas a las preferencias de los alumnos. Desde esa perspectiva ¿cómo debe ser la preparación de los docentes?

AS: Los docentes están en un permanente proceso de adaptación al 
cambio, aprendiendo y desarrollando nuevas metodologías y estrategias docentes que permitan a los alumnos aprender más y mejor. Lo que sucede es que los cambios se están sucediendo cada vez con mayor rapidez. Por ese motivo, la formación de los docentes debe ir evolucionando hacia su propia adaptación al cambio, con un mayor desarrollo de lo que denominan las "competencias suaves", que vayan en la línea de saber acompañar y guiar a sus alumnos en los objetivos y, eso sí, también hacerles ver cuándo se equivocan.

Es un error plantear que quien quiere o debe aprender ya lo sabe todo y los demás deben plegarse a sus demandas. Tiene que generarse un equilibrio en ese sentido. Los alumnos deben asumir también su rol de apoderamiento, pero con la modestia y la humildad como principios fundamentales. No aprende quien cree que ya tiene respuesta a todo.

\section{RS: ¿Están preparados los siste-} mas educativos para que la educación vaya más allá de la formación profesional y contemple las humanidades, la sociedad, la cultura, en resumen, "aprender a ser"?

AS: Este es el reto. El problema siempre está en que nos significamos hacia los extremos. Los sistemas educativos acostumbran a moverse bajo la "ley del péndulo".

Hoy estamos aquí, y como no sale todo lo bien como esperábamos, entonces nos vamos hacia el extre- mo contrario. La palabra, de nuevo, es "equilibrio". Por eso, cuando muchísima gente ha elevado el estandarte de la formación STEM (Ciencia, Tecnología, Ingeniería, Matemáticas), muchos pedimos incorporar ahí una "A" de artes, de humanismo, de cultura. STEAM va a formar personas mucho más completas que solo STEM. El "aprender a ser" significa que debemos enfocar la formación de las personas en su integralidad. Cualquiera, antes que ingeniera, doctor, científica o maestro, es persona. $Y$ ese debe ser el centro de nuestros sistemas educativos: formar personas que después sean capaces de decidir el ámbito de conocimiento en el que quieren desarrollar su vida profesional y que estén capacitados para crecer en él, con autonomía y autoregulación suficiente. $Y$ ese debe ser también el objetivo, de los docentes.

RS: Considerando las necesidades básicas insatisfechas de los individuos en países como el nuestro, en que la inequidad es evidente ¿educación y espacios laborales deberían ir de la mano? ¿Cómo? ¿Porqué?

AS: Es indudable que una mayor colaboración entre el mundo educativo y el laboral redundaría en beneficio del futuro de los alumnos, sin olvidar nunca el aspecto formativo integral al que me he referido anteriormente; es decir, el sistema educativo no debe formar solo para el trabajo, pero sin duda debe ayudar a ello, porque la dimensión pro- 
fesional también formará parte del "ser" de nuestros alumnos.

En este sentido, se está prodigando la tendencia que defiende la formación "dual", en la que institución educativa y empresa se coordinan para que los alumnos puedan aprender oficios y especialidades profesionales a la vez que asisten a los centros educativos.

Una parte de la formación se lleva a cabo en las empresas, bajo la supervisión de tutores conectados con el centro educativo. Es una línea de trabajo que en mi opinión tiene mucho futuro. Yo, personalmente, la he experimentado al más alto nivel en calidad de director del Plan de Doctorados Industriales del Gobierno de Cataluña, en el que se impulsan proyectos de colaboración público-privada entre grupos de investigación de las universidades y empresas que necesitan desarrollar una investigación estratégica para la innovación en su negocio. Existen también colaboraciones de este tipo a nivel de Maestría, así como en la Formación Profesional no universitaria.

\section{RS: ¿La lúdica debería ser una prioridad en el proceso educativo?}

AS: Más que la lúdica, es importante que aprender no sea sufrimiento, sino la celebración del descubrimiento de lo desconocido, de lo nuevo. Sin embargo, nuevas metodologías como la gamificación están abriendo un espacio importante en las nuevas formas de aprender, y es importante tenerlas en cuenta.

RS: La tecnología como protagonista de la educación actual, ¿qué requiere a su alrededor para garantizar resultados de inclusión y apropiación en el futuro inmediato?

AS: Nos encontramos ante tres brechas: la de acceso, la de uso y la apropiación. Necesitamos facilitar la conectividad de redes en todo el mundo, país por país; necesitamos poder dotar de conexiones a los alumnos, a los ciudadanos: yo abogo porque el acceso a Internet sea un derecho universal en estos momentos en que la mayor parte de la información circula por ese medio; necesitamos dotar de dispositivos a las personas para que las conexiones sean un hecho y no una teoría; y, finalmente, necesitamos desarrollar programas de alfabetización y capacitación digital para que su uso sea apropiado; a los docentes, por un lado, a los alumnos por otro, y a la ciudadanía en general por un tercero.

\section{RS: ¿Cómo define educación y emprendimiento digital?}

AS: Básicamente como la capacidad de poder gestionar la información que existe en las redes, de manera que sepamos adónde ir para encontrar lo que buscamos. Así mismo, cada uno debe convertirse en un procurador de nuevo contenido, para que existan interacciones y se genere en un modo positivo lo que Surowiecki denomina la "inteligencia de las multitudes". 
El emprendimiento es necesario para que nuevas ideas provenientes del juicio justo y ponderado, a la vez que creativo e imaginativo de los jóvenes -por eso es importante educarlos bien-, y de los mayores, también, de ahí que sea siempre importante contar con su experiencia.

RS: Los expertos hablan de la "metodología transmedia", en otras palabras, diferentes medios transmitiendo contenidos. En ese marco, el alumno por lo general utiliza solo un canal en la asimilación del conocimiento y siempre le faltará buena parte de la información. En su concepto, ¿qué se requiere para que pueda disponer del mensaje completo?

AS: Hoy en día creo que el alumno ya utiliza más de un canal. Mi investigación sobre "ecologías de aprendizaje" me demuestra que cada vez más, las decisiones que toman las personas para aprender se mueven en un marco en el que tenemos el aprendizaje formal, no formal e informal en un eje; y las modalidades presencial, híbrida o blended y online en el otro. Lo que es importante es que los docentes y las instituciones entiendan este marco y generen oportunidades de aprendizaje en otros cuadrantes del mismo, para que puedan llegar a los alumnos que utilizan canales distintos para aprender y actualizarse.

RS: Considerando la infraestructura educativa como un hecho, en términos de hardware y software, los contenidos cobran muchísima fuerza y en varios formatos (videos, información web, podcasts, para citar algunos). ¿Estos aspectos deberían ser prioritarios en las políticas de Estado en el mundo?

AS: Eso es solo una parte. El error que siempre se comete es atacarlo desde perspectivas independientes, cuando el mejor ataque sería el integral. En nuestra sociedad actual, encontrar contenidos no es difícil. Lo difícil es saber si son los adecuados. Por eso defiendo el rol de los docentes ante supuestos modelos de autoaprendizaje que, si bien deben existir, no son los que van a capacitar mejor a los alumnos. Es el acompañamiento de los docentes, su orientación y guía, lo que va a hacer que los alumnos puedan convertir la información en conocimiento. Igualmente, cabe promover el trabajo en equipo y el aprendizaje colaborativo. Los iguales nos enseñan muchas cosas, especialmente si somos conscientes de que formamos parte de una comunidad de aprendizaje en la que el todo es superior a la suma de las partes.

Sin embargo, sí. La infraestructura educativa y los contenidos deberían formar parte, también, de las prioridades en las políticas de los Estados.

RS: Desde mucho antes de la pandemia, varios expertos vaticinaban que la educación sería tridimensional y virtual; es decir, el conoci- 
miento disponible en vivo y los alumnos interactuando en entornos simulados. Hoy es una realidad. ¿Cuáles acciones son necesarias por parte de los dirigentes en ese contexto?

AS: Esa es una pregunta muy compleja. La tecnología siempre avanza a una velocidad a la que no es posible acogerse para que el conjunto de la ciudadanía pueda disfrutar de ella, porque existen velocidades muy diferentes en nuestra sociedad.

Es cierto que una de las líneas más claras de futuro, en este caso, un futuro cercano, es la del uso de la realidad virtual para la educación y la formación. Esto nos tendrá que ayudar a superar las dificultades que tenemos cuando queremos integrar la vertiente práctica de un aprendizaje. Es importante aceptar que se puede aprender así, y empezar a invertir dinero en las instituciones y en los sistemas para que sea posible, para que sea realidad un aprendizaje más flexible, pero de la misma o superior calidad.
RS: En su opinión, ¿los modelos escolares vigentes soportan las necesidades actuales, producto del coronavirus?

AS: No lo sé, porque en los distintos países eso puede ser bien distinto. Pero en general, creo que no. Hay unas asunciones asumidas que no funcionan: pensar que solo con asistir a clase se aprende, que la interacción no puede realizarse si uno no ve al otro en la sincronía, la absoluta obsolescencia de los mecanismos de evaluación... Los modelos escolares deben evolucionar hacia modelos híbridos que equilibren bien el uso de la presencialidad y la no presencialidad, y de la sincronía y la asincronía. Modelos que permitan hacer crecer a los alumnos en su autonomía y capacidad de auto-regulación; modelos que disocien el tiempo de aprender del tiempo de enseñar; $y$, sobre todo, modelos que diseñen la evaluación de manera formativa, continuada y diversificada. Aún estamos lejos de eso, a pesar de los esfuerzos de muchos profesionales que deben aplaudirse.

Sara Gallardo M. Periodista comunicadora, universidad Jorge Tadeo Lozano. Ha sido directora de las revistas Uno y Cero, Gestión empresarial y Acuc Noticias. Editora de Aló Computadores del diario El Tiempo. Redactora en las revistas Cambio 16, Cambio y Clase Empresarial. Coautora del libro "Lo que cuesta el abuso del poder". Ha sido corresponsal de la revista Infochannel de México; de los diarios La Prensa de Panamá y La Prensa Gráfica de El Salvador y corresponsal de la revista IN de Lanchile e investigadora en publicaciones culturales. Se ha desempeñado también como gerente de Comunicaciones y Servicio al Comensal en Inmaculada Guadalupe y amigos en Cía. S.A. (Andrés Carne de Res) y editora de Alfaomega Colombiana S.A. En la actualidad es asesora en escritura y producción de libros y editora de esta revista. 